\title{
Probe-dependent Dirac-point gap in the gadolinium-doped thallium-based topological insulator $\mathrm{TIBi}_{0.9} \mathrm{Gd}_{0.1} \mathrm{Se}_{2}$
}

\author{
S. O. Filnov ${ }^{1},{ }^{1,}$ I. I. Klimovskikh, ${ }^{1}$ D. A. Estyunin, ${ }^{1}$ A. V. Fedorov, ${ }^{2,3}$ V. Yu. Voroshnin, ${ }^{3,1}$ A. V. Koroleva, ${ }^{1}$ A. G. Rybkin, ${ }^{1}$ \\ E. V. Shevchenko, ${ }^{1}$ Z. S. Aliev ${ }^{0},{ }^{4,5}$ M. B. Babanly, ${ }^{6,7}$ I. R. Amiraslanov,${ }^{5,7}$ N. T. Mamedov, ${ }^{5}$ E. F. Schwier, ${ }^{8}$ K. Miyamoto, ${ }^{8}$ \\ T. Okuda, ${ }^{8}$ S. Kumar, ${ }^{8}$ A. Kimura, ${ }^{9,10}$ V. M. Misheneva, ${ }^{1}$ A. M. Shikin, ${ }^{1}$ and E. V. Chulkov ${ }^{11,12,1,13}$ \\ ${ }^{1}$ Saint Petersburg State University, 198504 Saint Petersburg, Russia \\ ${ }^{2}$ IFW Dresden, Helmholtzstrasse 20, 01069 Dresden, Germany \\ ${ }^{3}$ Helmholtz-Zentrum Berlin fr Materialien und Energie, Albert-Einstein-Strasse 15, 12489 Berlin, Germany \\ ${ }^{4}$ Azerbaijan State Oil and Industry University, AZ 1010 Baku, Azerbaijan \\ ${ }^{5}$ Institute of Physics, Azerbaijan National Academy of Sciences, AZ 1143 Baku, Azerbaijan \\ ${ }^{6}$ Institute of Catalysis and Inorganic Chemistry, Azerbaijan National Academy of Science, AZ1143 Baku, Azerbaijan \\ ${ }^{7}$ Baku State University, AZ 1148 Baku, Azerbaijan \\ ${ }^{8}$ Hiroshima Synchrotron Radiation Center, Hiroshima University, 2-313 Kagamiyama, Higashi-Hiroshima, Hiroshima 739-0046, Japan \\ ${ }^{9}$ Department of Physical Sciences, Graduate School of Science, Hiroshima University, 1-3-1 Kagamiyama, \\ Higashi-Hiroshima, Hiroshima 739-8526, Japan \\ ${ }^{10}$ Graduate School of Advanced Science and Engineering, Hiroshima University, 1-3-1 Kagamiyama, Higashi-Hiroshima 739-8526, Japan \\ ${ }^{11}$ Tomsk State University, 634050 Tomsk, Russia \\ ${ }^{12}$ Donostia International Physics Center (DIPC), 20018 San Sebastin/Donostia, Basque Country, Spain \\ ${ }^{13}$ Centro de Física de Materiales (CFM-MPC), Centro Mixto CSIC-UPV/EHU, 20080 San Sebastin/Donostia, Basque Country, Spain
}

(Received 31 December 2019; revised 11 June 2020; accepted 27 July 2020; published 26 August 2020)

\begin{abstract}
A tunable gap in the topological surface state is of great interest for novel spintronic devices and applications in quantum computing. Here, we study the surface electronic structure and magnetic properties of the Gd-doped topological insulator $\mathrm{TlBi}_{0.9} \mathrm{Gd}_{0.1} \mathrm{Se}_{2}$. Utilizing superconducting quantum interference device magnetometry, we show paramagnetic behavior down to $2 \mathrm{~K}$. Combining spin- and angle-resolved photoemission spectroscopy with different polarizations of light, we demonstrate that the topological surface state is characterized by the Dirac cone with a helical spin structure and confirm its localization within the bulk band gap. By using different light sources in photoemission spectroscopy, various Dirac-point gap values were observed: $50 \mathrm{meV}$ for $h v=18 \mathrm{eV}$ and $20 \mathrm{meV}$ for $h v=6.3 \mathrm{eV}$. Here, we discuss the gap observation by the angle-resolved photoemission spectroscopy method as a consequence of the scattering processes. Simulating the corresponding spectral function, we demonstrate that the asymmetric energy-distribution curve of the surface state leads to an overestimation of the corresponding gap value. We speculate that $20 \mathrm{meV}$ in our case is a trustworthy value and attribute this gap to be originated by scattering both on magnetic and charge impurities provided by Gd atoms and surface defects. Given the complexity and importance of scattering processes in the topological surface state together with our observations of distinctive photoemission asymmetry, we believe our results are important for research of the massive Dirac fermions in novel quantum materials.
\end{abstract}

DOI: 10.1103/PhysRevB.102.085149

\section{INTRODUCTION}

To date, many breakthroughs have been achieved in the field of topologically nontrivial materials. The most exciting ones are the theoretical classification and experimental realization of topological insulators (TIs) [1-4]. These bulk insulating materials host a metallic surface state, i.e., the socalled topological surface state (TSS) described by the Dirac cone. A TSS is characterized by spin-momentum locking and time-reversal symmetry (TRS), which largely suppress backscattering. In the heart of such electronic structure lies band inversion at the $\bar{\Gamma}$ point originated from strong spin-orbit coupling (SOC).

\footnotetext{
*st040513@student.spbu.ru
}

Among others, investigation of the interaction between the TSS and a magnetic field is of great interest. Magnetic order breaks TRS, lifting the Kramers degeneracy between opposite spin-oriented electronic states, which leads to a gap opening at the Dirac point. Theoretically, it is predicted that such behavior of the electronic structure can produce many fundamental effects. The most remarkable are the realization of topological magnetoelectric effect [5], quantum anomalous Hall (QAH) effect [6,7], and observation of magnetic monopoles [8]. Furthermore, there are many concepts of spintronics devices based on magnetic TIs, e.g., spin transistors [9].

Possible ways to create a magnetic order in TIs are doping by transition [10-14] or rare-earth metals [15-17], proximity effect with magnetic layer [18], or growing stoichiometric magnetic systems $[19,20]$. Thus, studies of chromiumdoped [11] and vanadium-doped [13] TIs made it possible 
to achieve the QAH effect at extremely low temperatures of 30 and $130 \mathrm{mK}$, respectively. Investigation of the manganesebased stoichiometric TI $\mathrm{MnBi}_{2} \mathrm{Te}_{4}$ revealed antiferromagnetic order below $25 \mathrm{~K}$ and a large magnetic gap at the Dirac point [19], as well as a transition to the QAH regime at a much higher temperature of $6.5 \mathrm{~K}$ [21]. Nevertheless, unambiguous magnetic-driven behavior was observed only for Rashba and bulk conduction-band states [22], while the Dirac cone state seemed to be unchanged under a magnetic transition. Therefore, discussions about the existence of the gap at the Dirac point and its nature have been part of most investigating topics in the TI research area in past years. In addition to the magnetic mechanism of the gap opening, there are other nonmagnetic mechanisms, e.g., impurity scattering on nonmagnetic atoms [23], scattering on the potential of magnetic atoms [24,25], or even a Higgs-like mechanism [26], that can open a gap at the Dirac point. However, such a gap has a nonmagnetic origin and does not lead to the observation of the aforementioned effects.

Our study is focused on the investigation of the electronic structure and magnetic properties of the gadolinium (Gd)-doped topological insulator $\mathrm{TlBi}_{0.9} \mathrm{Gd}_{0.1} \mathrm{Se}_{2}$. Previously, Gd-doped $\mathrm{Bi}(\mathrm{Sb})_{2} \mathrm{Se}(\mathrm{Te})_{3}$-like TIs were studied, demonstrating antiferromagnetic $[17,27,28]$ or paramagnetic [29] order. In Ref. [28], we observed that the Gd-doped TI $\mathrm{Bi}_{1.09} \mathrm{Gd}_{0.06} \mathrm{Sb}_{0.85} \mathrm{Te}_{3}$ has unique magnetic properties, with a ferromagnetic hysteresis loop at $100 \mathrm{~K}$ which disappears with a decrease of temperature. This effect can be related to the two-dimensional surface magnetism via a TSS RudermanKittel-Kasuya-Yosida (RKKY)-like mechanism [30]. However, we did not observe a significant gap at the Dirac point by angle-resolved photoemission spectroscopy (ARPES), which is possibly related to a small percentage of magnetic doping. Thus, the investigation of Gd-doped TIs is important to understand the impact of the magnetic atoms on a TI's electronic structure.

The undoped compound $\mathrm{TlBiSe}_{2}$ was theoretically $[31,32]$ and experimentally [33] investigated, demonstrating a promising electronic structure with the linearly dispersing surface state within the bulk band gap. A distinctive feature of thallium-based TIs is the absence of a van der Waals space between blocks of atomic layers, which can affect the magnetic properties in the case of doping by magnetic atoms.

Here, we present the investigation of the electronic structure and magnetic properties of the Gd-doped thallium-based topological insulator with stoichiometry $\mathrm{TlBi}_{0.9} \mathrm{Gd}_{0.1} \mathrm{Se}_{2}$ by means of angle- and spin-resolved photoemission spectroscopy and superconducting quantum interference device (SQUID) magnetometry. Special attention will be paid to the Dirac point gap analysis in the photoemission spectra.

\section{EXPERIMENTAL METHODS}

The $\mathrm{TlBi}_{0.9} \mathrm{Gd}_{0.1} \mathrm{Se}_{2}$ compound was synthesized by melting under the vacuum conditions of the elements of $\mathrm{Tl}, \mathrm{Bi}$, $\mathrm{Gd}$, and Se taken in an amount corresponding to the chemical formula. The crystal structure was refined by the Rietveld method based on the powder diffraction data (see Supplemental Material at [34]). Investigation of the magnetic properties of the sample was carried out at the Center for Diagnostics of
Materials for Medicine, Pharmacology and Nanoelectronics, Saint Petersburg State University Research Park, using the SQUID magnetometer with a helium cryostat manufactured by Quantum Design. The measurements were carried out in a "pull" mode in terms of temperature and magnetic field. The applied magnetic field was perpendicular to the sample surface (0001). The electronic structure of the surface state was studied using the ARPES method. The ARPES data were taken at the UE-112 beam line $1^{3}$ endstation of the BESSY-II synchrotron radiation facility in Berlin, Germany, BL-9, and Laser-ARPES stations at the Hiroshima Synchrotron Radiation Center, Japan. Spin-resolved photoemission spectroscopy (spin-ARPES) was measured at the BL-9B using a VLEED detector. The measurements were made at the $\bar{\Gamma}$ point of the Brillouin-zone projection on the surface (0001) using different photon energy. The photoemission spectra were collected on freshly cleaved surfaces of crystals at a temperature of $15 \mathrm{~K}$. Energy distribution curves (EDCs) shown in Fig. 4 were fitted using a composition of Voight and sigmoid functions $F(E)=V(E) \cdot \gamma(E) ; V(E)=L(E) * G(E)$, where $L(E)$ is a Cauchy-Lorentz distribution and $G(E)$ is a Gaussian distribution; and $\gamma(E)=\frac{2 \gamma_{0}}{1+\exp a\left(E-E_{0}\right)}$, where $E_{0}$ is peak position, $\gamma$ is the full width at the half maximum of the peak, and $a$ is the asymmetry. Spin-resolved spectra $\left(I_{\text {up }}, I_{\text {down }}\right)$ are plotted as $I_{\text {up } / \text { down }}=\frac{(1 \pm P)\left(I_{+}+I_{-}\right)}{2}, P=\frac{1}{S} \frac{\left(I_{+}-I_{-}\right)}{\left(I_{+}+I_{-}\right)}$, where $I_{+}$and $I_{-}$are the intensity of the reflected electrons by a positively and negatively magnetized target, respectively, and $S$ is the Sherman function. Circular dichroism (CD)-ARPES spectra are presented as $I=N_{+}-N_{-}$(where $N_{+}$and $N_{-}$, respectively, are the ARPES intensities for positive and negative circular polarized light). CD-ARPES images with a more familiar presentation of intensity as $I=\frac{\left(N_{+}-N_{-}\right)}{\left(N_{+}+N_{-}\right)}$are shown in the Supplemental Material [34].

\section{RESULTS}

\section{A. Magnetic properties}

To study the magnetic properties of the compound, we carried out the SQUID magnetometry measurements. In Fig. 1(a), one can see magnetization per mole presented as a function of the applied out-of-plane magnetic field up to $\pm 5 \mathrm{~T}$ [perpendicular to the sample's cleavage plane (0001)] at different temperatures from 2 to $300 \mathrm{~K}$. The isothermal magnetization vs magnetic field measured at $T=2 \mathrm{~K}$ (blue) has the well-known S-like shape that is typical for paramagnetic behavior. The temperature dependence of magnetic susceptibility $(\chi)$ in the applied out-of-plane field of $H=$ $0.1 \mathrm{~T}$ is presented in Fig. 1(b), together with $1 / \chi(T)$ in the inset. One can see the approximation (red dashed line) of the dependence by the Curie-Weiss law $\chi=\frac{C}{(T-\Theta)}$, where $C$ is the material-specific constant and $\Theta$ is the Weiss temperature. Fitting of the experimental points with Curie-Weiss law gives the Weiss temperature $\Theta \sim 0$, which points to the paramagnetic order in the whole accessible temperature range. Such paramagnetic behavior was also observed for Cr-doped $\mathrm{TlBiSe}_{2}$ [35].

From the fitting parameters of $1 / \chi(T)$, the reasonable, effective magnetic moment of $\mu_{\mathrm{eff}}=8.0 \mu_{\mathrm{B}}$ per Gd atom was found. This $\mu_{\text {eff }}$ is equal to the theoretical value of $8.0 \mu_{\mathrm{B}}$ 

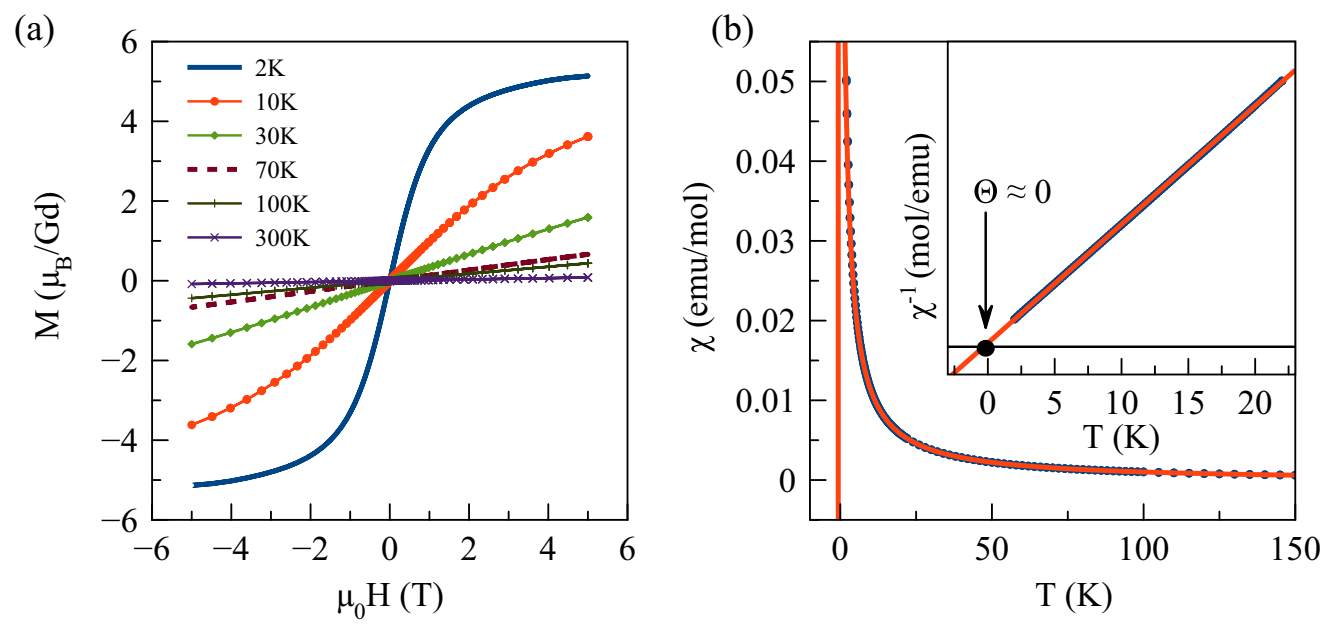

FIG. 1. (a) Magnetic field dependencies of the effective magnetization at different temperatures. (b) Temperature dependence of the magnetic susceptibility and inverse temperature dependence of the magnetic susceptibility of the sample.

expected for free $\mathrm{Gd}^{3+}$ ions [36]. These results indicate that $\mathrm{Gd}$ atoms effectively substitute for $\mathrm{Bi}$ atoms.

\section{B. Electronic and spin structure of the topological surface state}

\section{Angle-and spin-resolved photoemission measurements of the topological surface state}

To study the electronic structure of the TSS in $\mathrm{TlBi}_{0.9} \mathrm{Gd}_{0.1} \mathrm{Se}_{2}$, we carried out ARPES measurements with photoexcitation by laser and synchrotron radiation. In Figs. 2(a) and 2(b), ARPES spectra measured by using a laser radiation at the $\bar{\Gamma}$ point and near the Fermi level are shown. One can see that the electronic structure is characterized by a cone-shape state with a crossing point near $0.4 \mathrm{eV}$ relative to the Fermi level. Furthermore, we estimate the bulk band gap to be around $0.3 \mathrm{eV}$, which is the same as for pure $\mathrm{TlBiSe}_{2}$ [33]. (a)

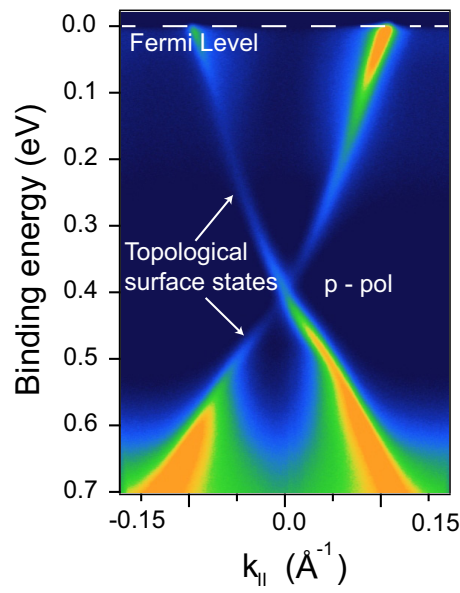

(b)

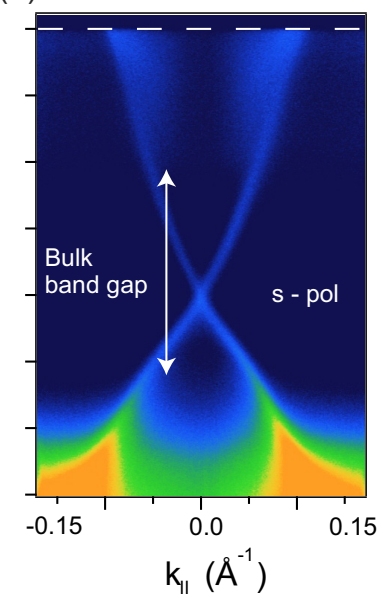

FIG. 2. ARPES intensity map as a function of binding energy and momentum for $\mathrm{TlBi}_{0.9} \mathrm{Gd}_{0.1} \mathrm{Se}_{2}$ measured at the $\bar{\Gamma}$ point with (a) $p$ and (b) $s$ polarizations of light. The photon energy is $6.3 \mathrm{eV}$ and the temperature is $15 \mathrm{~K}$.
Additionally, we carried out circular dichroism angleresolved photoemission spectroscopy (CD-ARPES) measurements. Measurements with different circular polarization can highlight the variation in the total orbital momentum of states, and in the case of the strong spin-orbit coupled Dirac cone state, it may highlight the spin texture [37]. In Figs. 3(a) and 3(b), CD-ARPES spectra for different photon energies are shown. One can see a complex CD signal structure as well as the difference between laser and synchrotron radiation-based CD-ARPES images. In Fig. 3(a), the sign of the CD signal changes several times in the valence-band region. Moreover, it is surprising to see the same sign for $\mathrm{k}^{+}$and $\mathrm{k}^{-}$for the Dirac cone branches slightly above the Dirac point despite the helical spin structure of the TIs in the initial state [1].

These features can be explained by a strong final-state effect in the case of low-energy radiation. In Refs. [38,39], it was shown that energy and polarization of light have a strong impact on spin-ARPES and CD-ARPES measurements. It was observed that by using light with energy of $6 \mathrm{eV}$, the photoemission process can switch the spin orientation between the in plane and out of plane, whereas using higher photon energies allows one to probe the initial TSS spin texture of $\mathrm{Bi}_{2} \mathrm{Se}_{3}$. To reduce the impact of the final-state effect, we carried out high-energy CD-ARPES measurements using $18 \mathrm{eV}$ photoexcitation light, shown in Fig. 3(b). One can see that near the Dirac point, $\mathrm{k}^{+}$and $\mathrm{k}^{-}$states have opposite $\mathrm{CD}$ signal, which points toward the helical spin structure of the state. In addition, two bands in the valence-band region are still seen, but with a bit higher binding energy compared to the laser-based one. Also, the CD sign of the upper cone changes at $0.3 \mathrm{eV}$ binding energy, which is possibly a result of the interaction between the TSS and conduction band. One can see that the data obtained with the $18 \mathrm{eV}$ photon energy look more symmetric and better correspond to the spin structure of the TIs in the initial state compared to the laser-radiationbased one.

In order to study the spin structure directly [Figs. 3(c) and 3(d)], we carried out spin-ARPES measurements for the in-plane component of the spin (the spin direction is along the 
(a)

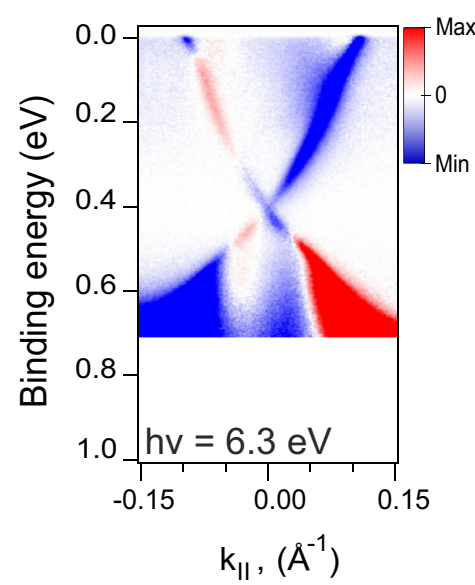

(b)

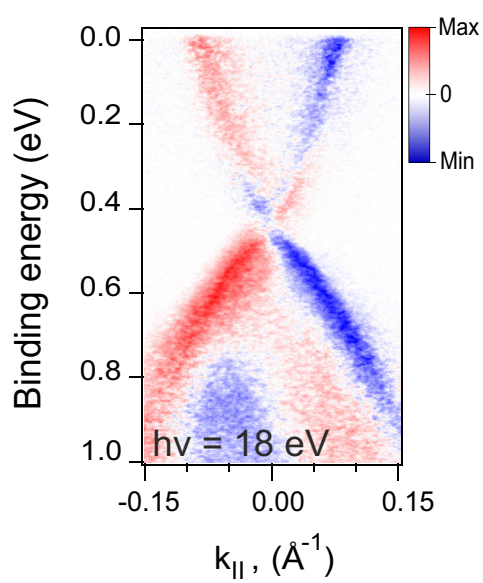

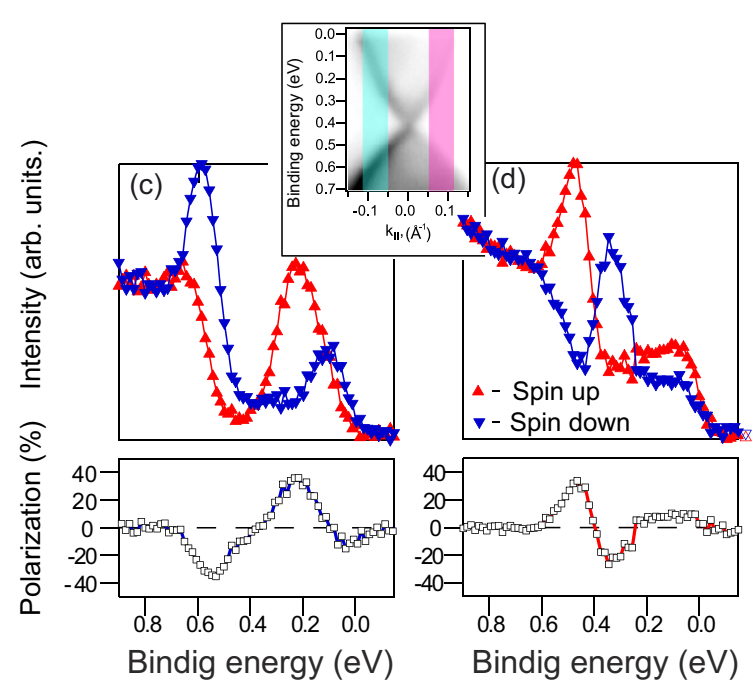

FIG. 3. (a),(b) Images of CD-ARPES measurements carried out using laser and synchrotron radiation with the shown energies. (c),(d) SpinARPES measurements of the Dirac cone branches. ARPES image with momentum regions marked by cyan and magenta stripes in which the spin spectra were measured is shown in the insert.

sample surface and perpendicular to the wave vector). One can see that the sign of spin polarization changes upon crossing the Dirac point, which is located at $0.4 \mathrm{eV}$ relative to the Fermi level (see ARPES inset). The spin polarization of the Dirac cone branches reaches the value of almost $40 \%$, which is consistent with that for the pristine compound [40]. Therefore, by ARPES, CD-ARPES, and spin-ARPES methods, we demonstrate that the electronic structure of $\mathrm{TlBi}_{0.9} \mathrm{Gd}_{0.1} \mathrm{Se}_{2}$ near the Fermi level is characterized by the cone-shaped surface electronic state with the helical spin structure located within the bulk band gap in the vicinity of the $\bar{\Gamma}$ point. These facts prove that $\mathrm{TlBi}_{0.9} \mathrm{Gd}_{0.1} \mathrm{Se}_{2}$ is a three-dimensional (3D) topological insulator with the Dirac-cone-like topological surface state.

\section{Energy-dependent features of the topological surface state}

In Fig. 4(a), a laser-based ARPES spectrum measured with $s$-polarized laser radiation with analysis of the Dirac cone electronic structure near the $\bar{\Gamma}$ point $(h v=6.3 \mathrm{eV})$ is presented. At the right part of Fig. 4(a), the second derivative plot $\left(d^{2} N / d E^{2}\right)$ is shown. One can see a small gap at the Dirac point in the second derivative image. To study the gap quantitatively, we analyze the energy distribution curve (EDC) at the $\bar{\Gamma}$ point shown by the red line in both the ARPES and second derivative image. Despite the absence of an intensity dip in the EDC at the Dirac point, a small asymmetry of the peak is seen. Fitting EDC with the sum of two Voight functions near the Dirac point (see the second derivative plot), we estimate the gap to be $20 \mathrm{meV}$.

Using the same analytical procedure, we treat the TSS electronic structure measured with $s$-polarized synchrotron radiation; see Fig. 4(b). Surprisingly, the size of the gap in this case reaches $50 \mathrm{meV}$ according to our estimations. It should be noted that all spectra were obtained using the same procedure that includes the preliminary measurements of $E\left(k_{x}, k_{y}\right)$ dispersion maps as a set of $E\left(k_{x}\right)$ at various $k_{y}$. This excludes misalignment along the $k_{y}$ direction (see Supplemental Material at [34]).

\section{DISCUSSION}

We assume that such difference of the Dirac-point gap size can be explained by the scattering and other final-state effects in the photoemission process. It is known that surface conditions of the solid are important for the electronic structure of the surface state. For example, it was shown that a decrease of terrace widths of the $\mathrm{Cu}(111)$ surface leads to asymmetry and broadening of the surface state peak [41,42].

It is evident that the synchrotron-radiation-based image of the TSS is much broader compared to the one measured with the laser radiation, and the corresponding EDC of the TSS at the Dirac point has a quite asymmetric shape.

Despite the difference in the excitation energy (and, correspondingly, kinetic energy of the photoelectrons), the difference between the laser and synchrotron stations used in the experiment is the light-spot profile. For laser ARPES measurements in Fig. 4(a), the light spot was $\sim 5 \times 5 \mu \mathrm{m}$ [43], while for the synchrotron-radiation-based one in Fig. 4(b), it was $\sim 20 \times 100 \mu \mathrm{m}$. Larger spot size may lead to an increase of the surface defects' influence on the spectra. To demonstrate how asymmetry modifies the size of the gap detected by ARPES, we simulated a spectral function using the band structure extracted from Fig. 4(a). Based on a quite symmetric EDC profile away from the Dirac point, we fit the surface state and present the corresponding peak maximum positions in Fig. 5(a). In Fig. 5(b), we reconstruct the laser-based image without a photoemission cross section and taking into account the exaggerated angular integration of $\pm 0.5^{\circ}$ perpendicular to the electron analyzer entrance slit. As the next step, in Fig. 5(c), we show the calculation using the same parameters of the peak width and angular integration as for the ARPES experiment in Fig. 4(b). One can see that it does not much change the gap value extracted by the standard fit procedure of the corresponding EDC as presented in Fig. 5(c). Finally, in Fig. 5(d), we show calculations of the spectral function using the asymmetric EDC profile, mimicking one experimentally observed away from the Dirac point in Fig. 4(b). It is clearly 
(a)

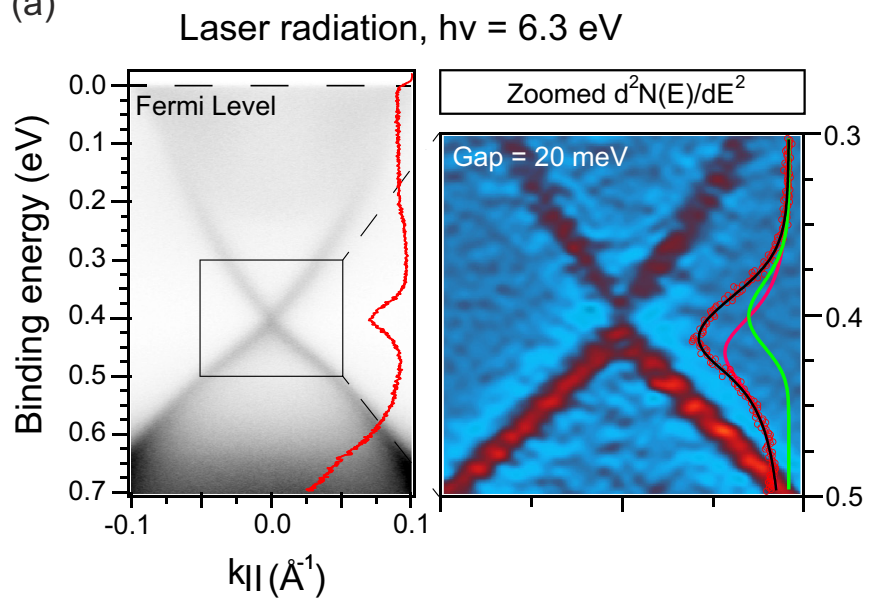

(b)

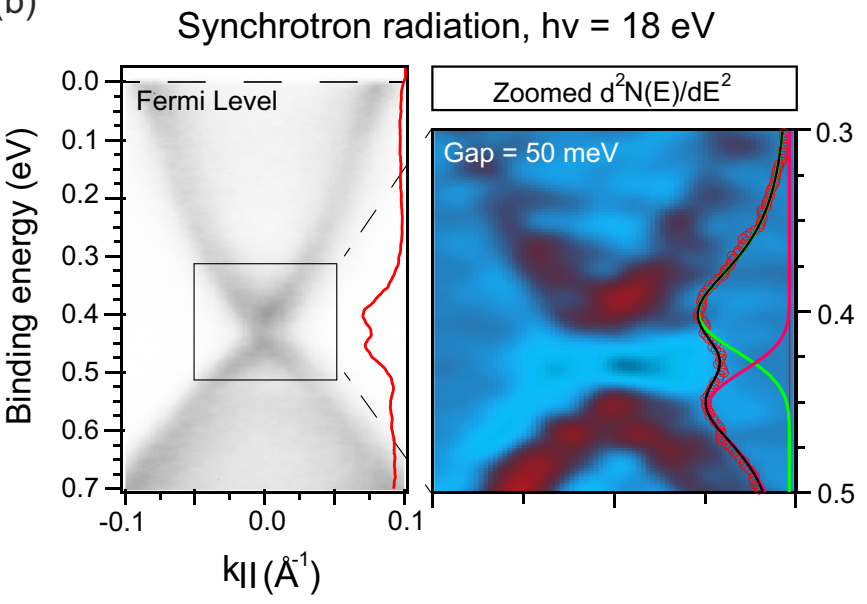

FIG. 4. (a) ARPES images of the $\mathrm{TlBi}_{0.9} \mathrm{Gd}_{0.1} \mathrm{Se}_{2}$ measured at the $\bar{\Gamma}$ point using laser radiation. The photon energy is $6.3 \mathrm{eV}$ and the temperature is $15 \mathrm{~K}$. (b) The same type of image, but measured by using synchrotron radiation. The photon energy is $18 \mathrm{eV}$ and the temperature is $15 \mathrm{~K}$. Near the ARPES images, the second derivative zoomed at the Dirac-point region is shown. Energy distribution curves at the Dirac point are presented by a red line. Decomposition of the EDC at the Dirac point is shown as pink and green lines. The resulting line is shown in black.

seen that the corresponding EDC profile through the Dirac point in Fig. 5(c) shows a presence of two peaks, while even with a resolution of the laser-based ARPES in Fig. 5(b) the energy gap was already invisible to the naked eye. Using the standard fit procedure, we extracted the gap size of about $55 \mathrm{meV}$, which is close to the one we extracted from the experimental data. Therefore, the Dirac-point (DP) gap of $20 \mathrm{meV}$, observed in Fig. 4(a), is a more trusted value. Its origin is possibly related to the impact of short-range magnetism by $\mathrm{Gd}^{3+}$ ions in the absence of magnetic ordering, while its increase up to $50 \mathrm{meV}$, shown in Fig. 4(b), is rather related to the impact of the photoemission process and scattering on surface defects.

The asymmetry presented in the photoemission spectra is a broadly studied phenomenon and it has many different origins. It appears as a fingerprint of photoelectron scat- tering, photoelectron diffraction, or the result of quantum interference with the continuum wave function (Fano line shape [44]). Here, we suppose that the scattering plays a major role as it is strongly connected to a surface quality. The impact of scattering processes on the topological surface state has attracted great attention, both from the experimental and theoretical sides, while their mechanisms are still not fully unraveled. Recently, scattering on nonmagnetic charge impurities was theoretically studied and leads to depletion of the density of states at the Dirac point [23]. Also, it was experimentally demonstrated $[45,46]$ that an increase of the sulfur amount in $\operatorname{TlBi}\left(\mathrm{Se}_{x} \mathrm{~S}_{1-x}\right)_{2}$ leads to an unexpected Dirac-point gap opening. In our case, we also observe the energy gap; however, a similar size is predicted for a much higher concentration of charge impurities. In addition, we observe quite interesting asymmetry, which changes its sign (a)

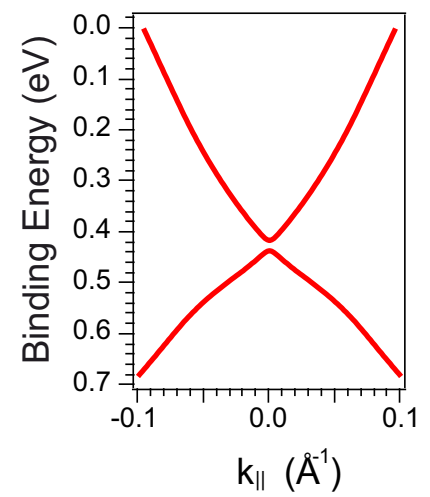

(b)

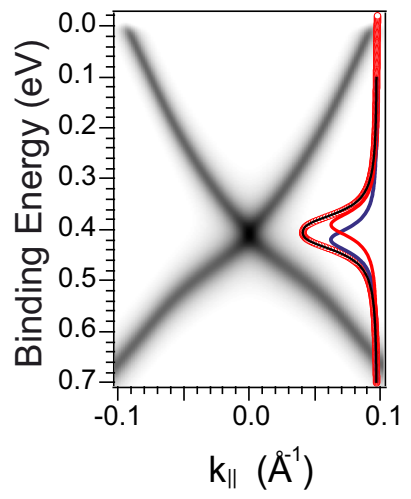

(c)

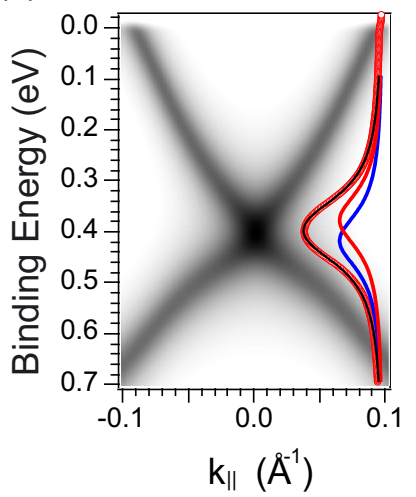

(d)

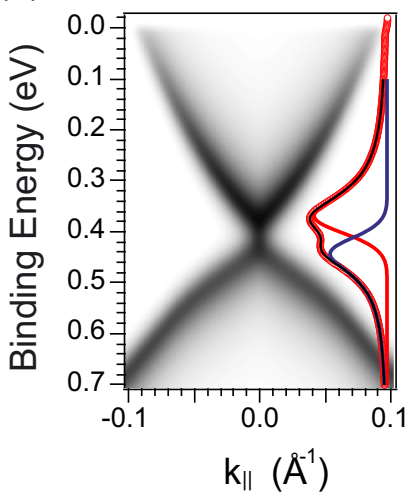

FIG. 5. Model of the ARPES spectral function mimicking experimental resolution. (a) Band structure fit of data from Fig. 4(a). (b) Spectral function for laser-based photoemission. (c) Spectral function for synchrotron-based photoemission. (d) Spectral function for synchrotron-based photoemission with the asymmetric EDC line profile. 
crossing the Dirac point. Such a behavior is not typical for photoemission in conventional materials and may help to trace exotic phenomena in topological insulators.

\section{CONCLUSION}

We have experimentally studied the electronic structure and magnetic properties of the Gd-doped topological insulator $\mathrm{TlBi}_{0.9} \mathrm{Gd}_{0.1} \mathrm{Se}_{2}$. SQUID measurement reveals paramagnetic order down to $2 \mathrm{~K}$. The electronic structure near the Fermi level measured by means of angle- and spin-resolved photoemission spectroscopy is characterized by the Dirac cone with a helical spin structure. Analysis of the Dirac cone at the $\bar{\Gamma}$ point demonstrates the Dirac-point gap opening, even in the paramagnetic phase. We observe the energy gap of $20 \mathrm{meV}$ at the Dirac point using laser-radiation-based ARPES and its increase up to $50 \mathrm{meV}$ using synchrotron-radiation-based ARPES. By simulation of ARPES spectra, we demonstrate that such a change is the result of the asymmetric shape of the photoemission spectrum presented in the synchrotron data. We assume that the possible origin of this asymmetry is the result of scattering processes on the surface of a solid state, but features of this phenomenon require further investigation.

\section{ACKNOWLEDGMENTS}

This work was supported by St. Petersburg State University Project (ID No. 51126254), by the Russian Science Foundation (Grant No. 18-12-00062), by the Ministry of Science and Higher Education of the Russian Federation (Grant No 20201902-01-058), and by the Science Development Foundation under the President of the Republic of Azerbaijan (Grant No. EIF-BGM-4-RFTF-1/2017-21/04/1-M-02). The studies were also carried out at the resource centers of St. Petersburg State University "Physical Methods for Surface Investigation" and "Diagnosis of Functional Materials for Medicine, Pharmacology, and Nanoelectronics." In addition, the work was supported by the German-Russian Interdisciplinary Science Center (G-RISC) funded by the German Federal Foreign Office via the German Academic Exchange Service (DAAD) and Russian-German Laboratory at BESSY II (HelmholtzZentrum, Berlin). We thank the Hiroshima Synchrotron Radiation Center (Proposal No. 18BG026), Helmholtz-Zentrum Berlin für Materialien und Energie for the allocation of synchrotron radiation beam times, and the N-BARD, Hiroshima University for supplying liquid helium. A.K. was financially supported by KAKENHI (Grants No. 17H06138, No. 17H06152, and No. 18H03683).
[1] M. Z. Hasan and C. L. Kane, Rev. Mod. Phys. 82, 3045 (2010).

[2] X.-L. Qi and S.-C. Zhang, Rev. Mod. Phys. 83, 1057 (2011).

[3] C. L. Kane and E. J. Mele, Phys. Rev. Lett. 95, 146802 (2005).

[4] L. Fu and C. L. Kane, Phys. Rev. B 76, 045302 (2007).

[5] T. Morimoto, A. Furusaki, and N. Nagaosa, Phys. Rev. B 92 , 085113 (2015).

[6] Q. L. He, L. Pan, A. L. Stern, E. C. Burks, X. Che, G. Yin, J. Wang, B. Lian, Q. Zhou, E. S. Choi, K. Murata, X. Kou, Z. Chen, T. Nie, Q. Shao, Y. Fan, S.-C. Zhang, K. Liu, J. Xia, and K. L. Wang, Science 357, 294 (2017).

[7] R. Yu, W. Zhang, H.-J. Zhang, S.-C. Zhang, X. Dai, and Z. Fang, Science 329, 61 (2010).

[8] X.-L. Qi, R. Li, J. Zang, and S.-C. Zhang, Science 323, 1184 (2009).

[9] M. Götte, M. Joppe and T. Dahm, Sci. Rep. 6, 36070 (2016).

[10] P. P. J. Haazen, J.-B. Laloë, T. J. Nummy, H. J. M. Swagten, P. Jarillo-Herrero, D. Heiman, and J. S. Moodera, Appl. Phys. Lett. 100, 082404 (2012).

[11] C.-Z. Chang, J. Zhang, X. Feng, J. Shen, Z. Zhang, M. Guo, K. Li, Y. Ou, P. Wei, L.-L. Wang, Z.-Q. Ji, Y. Feng, S. Ji, X. Chen, J. Jia, X. Dai, Z. Fang, S.-C. Zhang, K. He, Y. Wang, L. Lu, X.-C. Ma, and Q.-K. Xue, Science 340, 167 (2013).

[12] J. S. Dyck, P. Hájek, P. Lošt'ák, and C. Uher, Phys. Rev. B 65, 115212 (2002).

[13] C.-Z. Chang, W. Zhao, D. Y. Kim, H. Zhang, B. A. Assaf, D. Heiman, S.-C. Zhang, C. Liu, M. H. W. Chan, and J. S. Moodera, Nat. Mater. 14, 473 (2015).

[14] V. N. Antonov, L. V. Bekenov, S. Uba, and A. Ernst, Phys. Rev. B 96, 224434 (2017).

[15] S. E. Harrison, L. J. Collins-McIntyre, S. Li, A. A. Baker, L. R. Shelford, Y. Huo, A. Pushp, S. S. P. Parkin, J. S. Harris, E.
Arenholz, G. van der Laan, and T. Hesjedal, J. Appl. Phys. 115, 023904 (2014).

[16] S. E. Harrison, L. J. Collins-McIntyre, S.-L. Zhang, A. A. Baker, A. I. Figueroa, A. J. Kellock, A. Pushp, S. S. P. Parkin, J. S. Harris, G. van der Laan, and T. Hesjedal, J. Phys.: Condens. Matter 27, 245602 (2015).

[17] A. M. Shikin, D. A. Estyunin, Y. I. Surnin, A. V. Koroleva, E. V. Shevchenko, K. A. Kokh, O. E. Tereshchenko, S. Kumar, E. F. Schwier, K. Shimada, T. Yoshikawa, Y. Saitoh, Y. Takeda, and A. Kimura, Sci. Rep. 9, 4813 (2019).

[18] M. Li, Q. Song, W. Zhao, J. A. Garlow, T.-H. Liu, L. Wu, Y. Zhu, J. S. Moodera, M. H. W. Chan, G. Chen, and C.-Z. Chang, Phys. Rev. B 96, 201301(R) (2017).

[19] M. M. Otrokov, I. I. Klimovskikh, H. Bentmann, D. Estyunin, A. Zeugner, Z. S. Aliev, S. Gaß, A. U. B. Wolter, A. V. Koroleva, A. M. Shikin, M. Blanco-Rey, M. Hoffmann, I. P. Rusinov, A. Y. Vyazovskaya, S. V. Eremeev, Y. M. Koroteev, V. M. Kuznetsov, F. Freyse, J. Sánchez-Barriga, I. R. Amiraslanov, M. B. Babanly, N. T. Mamedov, N. A. Abdullayev, V. N. Zverev, A. Alfonsov, V. Kataev, B. Büchner, E. F. Schwier, S. Kumar, A. Kimura, L. Petaccia, G. Di Santo, R. C. Vidal, S. Schatz, K. Kißner, M. Ünzelmann, C. H. Min, S. Moser, T. R. F. Peixoto, F. Reinert, A. Ernst, P. M. Echenique, A. Isaeva, and E. V. Chulkov, Nature (London) 576, 416 (2019).

[20] M. M. Otrokov, I. P. Rusinov, M. Blanco-Rey, M. Hoffmann, A. Y. Vyazovskaya, S. V. Eremeev, A. Ernst, P. M. Echenique, A. Arnau, and E. V. Chulkov, Phys. Rev. Lett. 122, 107202 (2019).

[21] Y. Deng, Y. Yu, M. Z. Shi, Z. Guo, Z. Xu, J. Wang, X. H. Chen, and Y. Zhang, Science 367, 895 (2020).

[22] D. A. Estyunin, I. I. Klimovskikh, A. M. Shikin, E. F. Schwier, M. M. Otrokov, A. Kimura, S. Kumar, S. O. Filnov, Z. S. Aliev, 
M. B. Babanly, and E. V. Chulkov, APL Mater. 8, 021105 (2020).

[23] H. Liu, H. Jiang, Q.-f. Sun, and X. C. Xie, Phys. Rev. Lett. 113, 046805 (2014).

[24] A. M. Black-Schaffer, A. V. Balatsky, and J. Fransson, Phys. Rev. B 91, 201411(R) (2015).

[25] J. Sánchez-Barriga, A. Varykhalov, G. Springholz, H. Steiner, R. Kirchschlager, G. Bauer, O. Caha, E. Schierle, E. Weschke, A. A. Ünal, S. Valencia, M. Dunst, J. Braun, H. Ebert, J. Minár, E. Golias, L. V. Yashina, A. Ney, V. Holý, and O. Rader, Nat. Commun. 7, 10559 (2016).

[26] B. Yan, D. Zhang, and C. Felser, Phys. Stat. Solidi (RRL) 7, 148 (2013).

[27] J. Kim, K. Lee, T. Takabatake, H. Kim, M. Kim, and M.-H. Jung, Sci. Rep. 5, 10309 (2015).

[28] S. O. Filnov, Y. A. Surnin, A. V. Koroleva, I. I. Klimovskikh, D. A. Estyunin, A. Y. Varykhalov, K. A. Bokai, K. A. Kokh, O. E. Tereshchenko, V. A. Golyashov, E. V. Shevchenko, and A. M. Shikin, J. Expt. Theor. Phys. 129, 404 (2019).

[29] S.-W. Kim and M.-H. Jung, AIP Adv. 8, 101319 (2018).

[30] M. A. Ruderman and C. Kittel, Phys. Rev. 96, 99 (1954).

[31] S. V. Eremeev, G. Bihlmayer, M. Vergniory, Y. M. Koroteev, T. V. Menshikova, J. Henk, A. Ernst, and E. V. Chulkov, Phys. Rev. B 83, 205129 (2011).

[32] S. V. Eremeev, Y. M. Koroteev, and E. V. Chulkov, JETP Lett. 91, 594 (2010).

[33] K. Kuroda, M. Ye, A. Kimura, S. V. Eremeev, E. E. Krasovskii, E. V. Chulkov, Y. Ueda, K. Miyamoto, T. Okuda, K. Shimada, H. Namatame, and M. Taniguchi, Phys. Rev. Lett. 105, 146801 (2010).

[34] Please see Supplemental Material at http://link.aps.org/ supplemental/10.1103/PhysRevB.102.085149 for the compound characterization and the ARPES mapping data.

[35] Z. Wang, K. Segawa, S. Sasaki, A. A. Taskin, and Y. Ando, APL Mater. 3, 083302 (2015).
[36] S. E. Harrison, L. J. Collins-McIntyre, S. L. Zhang, A. A. Baker, A. I. Figueroa, A. J. Kellock, A. Pushp, Y. L. Chen, S. S. P. Parkin, J. S. Harris, G. van der Laan, and T. Hesjedal, Appl. Phys. Lett. 107, 182406 (2015).

[37] C. Liu and S. Zhang, in Topological Insulators, edited by M. Franz and L. Molenkamp, Contemporary Concepts of Condensed Matter Science Vol. 6 (Elsevier, New York, 2013), pp. 59-89.

[38] C. Seibel, J. Braun, H. Maaß, H. Bentmann, J. Minár, T. V. Kuznetsova, K. A. Kokh, O. E. Tereshchenko, T. Okuda, H. Ebert, and F. Reinert, Phys. Rev. B 93, 245150 (2016).

[39] J. Sánchez-Barriga, A. Varykhalov, J. Braun, S.-Y. Xu, N. Alidoust, O. Kornilov, J. Minár, K. Hummer, G. Springholz, G. Bauer, R. Schumann, L. V. Yashina, H. Ebert, M. Z. Hasan, and O. Rader, Phys. Rev. X 4, 011046 (2014).

[40] K. Kuroda, G. Eguchi, K. Shirai, M. Shiraishi, M. Ye, K. Miyamoto, T. Okuda, S. Ueda, M. Arita, H. Namatame, M. Taniguchi, Y. Ueda, and A. Kimura, Phys. Rev. B 91, 205306 (2015).

[41] A. Beckmann, K. Meinel, C. Ammer, M. Heiler, and H. Neddermeyer, Surf. Sci. 375, L363 (1997).

[42] A. Beckmann, K. Meinel, M. Heiler, C. Ammer, and H. Neddermeyer, Phys. Stat. Solidi (b) 198, 665 (1996).

[43] H. Iwasawa, E. F. Schwier, M. Arita, A. Ino, H. Namatame, M. Taniguchi, Y. Aiura, and K. Shimada, Ultramicroscopy 182, 85 (2017).

[44] A. L. Stancik and E. B. Brauns, Vib. Spectrosc. 47, 66 (2008).

[45] T. Sato, K. Segawa, K. Kosaka, S. Souma, K. Nakayama, K. Eto, T. Minami, Y. Ando, and T. Takahashi, Nat. Phys. 7, 840 (2011).

[46] S.-Y. Xu, M. Neupane, I. Belopolski, C. Liu, N. Alidoust, G. Bian, S. Jia, G. Landolt, B. Slomski, J. H. Dil, P. P. Shibayev, S. Basak, T.-R. Chang, H.-T. Jeng, R. J. Cava, H. Lin, A. Bansil, and M. Z. Hasan, Nat. Commun. 6, 6870 (2015). 